\title{
Carnets
}

Revue électronique d'études françaises de l'APEF

Première Série - 4 2012

(Res)sources de l'extravagance

\section{Une subjectivation extravagante. Métaphysique des tubes, d'Amélie Nothomb}

\section{Cristina Álvares}

\section{(2) OpenEdition}

\section{Journals}

\section{Édition électronique}

URL : http://journals.openedition.org/carnets/7083

DOI : 10.4000/carnets.7083

ISSN : 1646-7698

Éditeur

APEF

\section{Édition imprimée}

Date de publication : 1 janvier 2012

Pagination : 245-259

\section{Référence électronique}

Cristina Álvares, « Une subjectivation extravagante. Métaphysique des tubes, d'Amélie Nothomb», Carnets [En ligne], Première Série - 4 | 2012, mis en ligne le 20 juin 2018, consulté le 19 avril 2019. URL : http://journals.openedition.org/carnets/7083 ; DOI : 10.4000/carnets.7083

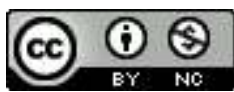

Carnets est mis à disposition selon les termes de la licence Creative Commons - Atribution - Pas d'utilisation commerciale 4.0 International. 


\title{
UNE SUBJECTIVATION EXTRAVAGANTE \\ Métaphysique des tubes, d'Amélie Nothomb
}

Cristina Alvares

Universidade do Minho

calvares@ilch.uminho.pt

\begin{abstract}
Résumé
Depuis Prétextat Tach jusqu'à Melvin Mapple, l'univers romanesque de Nothomb est peuplé d'obèses extravagants. Ces personnages sont du néant enveloppé de graisse, du néant qui prend de plus en plus de place, qui envahit, qui aspire. Cette invasion du vide qui est dans l'autre, qui constitue son noyau dur, sa force d'inertie, me gêne, m'angoisse, me dégoûte, est la thématique axiale de l'œuvre de Nothomb. Dans Métaphysique des tubes, le tube est la forme objectale du non-être. Ce roman raconte une subjectivation dont le moment crucial est celui où les carpes, des tubes enveloppés de graisse, donnent à voir à l'extérieur le noyau de non-être qui habite le sujet. Celui-ci se fonde du dégoût extravagant causé par l'extériorisation du tube interne.
\end{abstract}

\section{Abstract}

From Prétextat Tach to Melvin Mapple, Nothomb's fictional world is full of extravagantly obese characters. They are bits of nothingness surrounded by a fat substance, a nothingness that takes more and more room, invades and eventually consumes everything. The key subject of Nothomb's novels is this invasion of the self's space by another's emptiness, by their inert kernel of apathy, that disturbs, provokes anxiety and disgust to a point the self just can't stand it. In Métaphysique des tubes, the tube is the object that shapes the non-being. This novel tells the story of the construction of a subjective structure whose crucial moment is when the carps, tubes surrounded by fat, let the reader glimpse at the kernel of non-being inside the subject. The subject is founded by the extravagant disgust caused by the externalisation of the inner tube.

Mots-clés: ontologie, moi, sujet, dégoût, négatif

Keywords: ontology, ego, subject, disgust, negative 
Au tournant du millénaire, la publication de Stupeurs et tremblements en 1999 et de Métaphysique des tubes Métaphysique des tubes ${ }^{1}$ en 2000 semble avoir clôt une première période de la production littéraire d'Amélie Nothomb, qui avait débuté en 1992 avec Hygiène de l'assassin. L'enfance fantasmée comme stade mythique d'une ontologie sans faille est le thème des romans écrits entre 1992 et 1998 ainsi que l'obsession de leurs personnages mégalomanes et fétichistes qui souhaitent, par des moyens retors et pervers, soit la préserver, soit la retrouver. Prétextat Tach, Émile, Omer, Celsius: ces personnages sont des variations adultes sur le modèle de l'enfant divin au centre de son jardin nippon. En racontant la perte irréversible de la jouissance de l'être, Métaphysique des Tubes brise le stade du miroir et boucle ce premier ensemble romanesque centré sur le mythe de l'enfance, comme si l'auteure voulait en être quitte.

En tant que roman autobiographique, l'extravagance de Métaphysique des Tubes consiste à raconter la vie de la narratrice depuis l'état embryonnaire jusqu'à son troisième anniversaire. Cette période précoce de l'existence, avec les phases de son développement, est racontée dans le registre ontologique (métaphysique), combiné à une réécriture parodique du texte biblique, et aboutit à la subjectivation le jour du $3^{\mathrm{e}}$ anniversaire du personnage. La narratrice appelle ce qui s'est passé ce jour-là "son aventure fondatrice". II s'agit effectivement de la fondation de la subjectivité par un dégoût extravagant.

\section{Ontogenèse. Dieu et le tube}

La phrase inaugurale du roman est celle de la Genèse: "Au commencement il n'y avait rien" (Nothomb, 2000:5). Ce nihil originaire s'identifie à la plénitude de l'être dans l'absolue satisfaction et dans l'absolue indifférence. L'être à qui manque le manque est Dieu: "Il ne voulait rien, n'attendait rien, ne percevait rien, ne refusait rien et ne s'intéressait à rien". Sa satisfaction absolue est négative: sans objets et sans plaisirs, c'est la satisfaction de rien. Plein et dense comme un œuf dur, Dieu est l'être du nihil, d'un rien absolu qui n'est "ni vide ni vague", c'est-à-dire qui n'a pas de limite, de contour, de contraire. Dieu est tellement plein de rien qu'il nie la création: "Pour rien au monde il n'eût créé quoi que ce fût. Le rien faisait mieux que lui convenir: il le comblait”.

Ensuite Dieu prend une forme différente: d'œuf dur il passe à tube digestif (p.7) et le registre biologique vient se combiner au registre métaphysique. C'est là qu'est introduit le concept de tube, défini comme la forme objectale et sensible du négatif: "Les tubes sont de singuliers mélanges de plein et de vide, de la matière creuse, une membrane d'existence protégeant un faisceau d'inexistence" (2000:7). Ce rien entouré et limité par la forme de

\footnotetext{
${ }^{1}$ Toutes les citations sont tirées de cette édition.
} 
l'objet a perdu l'absolu et l'illimité du nihil génésique. L'association du tube à Dieu marque la première métamorphose de l'être: le tube digestif qu'est Dieu maintenant renvoie à un corps, lequel a la souplesse du tuyau, mais demeure rigide et inerte dans sa nature de tube. On comprend en lisant la suite que Dieu est, dans "la sérénité absolue du cylindre", un fœtus, puis un bébé dont l'être de tube se manifeste dans l'apathie et l'indifférence à la vie (la Plante). On comprend alors rétroactivement que l'œuf dur n'a pas seulement une signification métaphysique - Dieu-nihil - mais aussi une signification biologique - le zygote et désigne la cellule qui se déploiera en embryon. On comprend que cette morphogenèse se déploie autour du tube qui subsiste dans l'être comme noyau dur, reste du nihil d'où l'être a été tiré. II reste dans sa rigidité et son inertie de chose comme un noyau d'inorganique au sein de l'être vivant. Le tube est la chose qui reste de l'ex-nihilo de l'être.

Le récit poursuit en développant une théo-ontologie négative parodique comme métaphore de la croissance purement biologique du bébé. Le tube garde l'être dans l'indifférence au monde et à autrui et en fait la forme corporelle du 'néant qui prenait de plus en plus de place'. II garde son autarcie divine, l'immanence à son être même: "Manger ou ne pas manger, boire ou ne pas boire, cela lui était égal: être ou ne pas être, telle n'était pas sa question" (2000:11). L'être est ainsi un corps vivant très peu vivant dans son mutisme et son immobilité: "Les autres bébés faisaient leurs premiers pas, leurs premiers sourires, leurs premiers quelque chose. Dieu, lui, ne cessait d'effectuer son premier rien du tout" (2000:15). C'est la vie sans choix, sans refus, sans désir, sans plaisir. C'est le paradoxe de l'existence purement biologique qui se prolonge après la naissance - jusqu'à deux ans dans le cas du personnage - et qui est aussi inerte que celle d'un objet inorganique comme un tube: "Dieu était l'incarnation de la force d'inertie - la plus forte des forces. La plus paradoxale des forces, aussi: quoi de plus bizarre que cet implacable pouvoir qui émane de ce qui ne bouge pas? La force d'inertie, c'est la puissance du larvaire" (2000:13). C'est l'existence prépsychique et pré-sémantique de l'être sans langage, sans conscience, sans mémoire, sans "moi" et sans émoi. Certes, un tel prolongement est excessif et même contraire à l'expérience des bébés et des petits enfants dont l'état, loin de l'autarcie divine, est celui de l'ananké (la radicale dépendance à l'égard des soins procurés par autrui). Aussi, les bébés ne sont nullement indifférents aux êtres qui s'occupent d'eux et aux objets qui leur donnent du plaisir, en premier lieu le sein. Ils n'ont pas de moi mais ils ont des émois (et le fœtus de même, et surtout des émois sonores). Le prolongement exagéré de l'état intra-utérin jusqu'à deux ans marque le personnage du signe de l'extravagance pour en faire un être à part, unique et singulier (quelqu'un qui a gardé la condition divine-tubaire plus longtemps que les autres). Plus loin la narratrice identifie cet état à la mort et se définit en tant qu'enfant comme quelqu'un qui connaissait la mort mieux que le commun des mortels, non pas parce qu'elle serait héroïquement descendue aux enfers pour l'y affronter en combat, mais parce qu'elle 
avait mis deux ans pour sortir de la torpeur fœtale, cette lenteur s'inscrivant néanmoins dans le registre héroïque de l'hubris:

Mort! Qui mieux que moi savait? Le sens de ce mot, je venais à peine de le quitter! Je le connaissais encore mieux que les autres enfants, moi qui l'avais prolongé au-delà des limites humaines. N'avais-je pas vécu deux années de coma, pour autant que l'on puisse vivre le coma? Qu'avaient-ils donc pensé que je faisais dans mon berceau, pendant si longtemps, sinon mourir ma vie, mourir le temps, mourir la peur, mourir le néant, mourir la torpeur? (2000:46; je souligne).

\section{La formation du moi dans le plaisir et l'avènement de l'enfant divin}

L'étape suivante est liée à l' "accident cérébral" qui a mis fin à l'autarcie et à l'apathie du bébé. Cet accident déclenche "la sainte colère de Dieu" qui exprime sa haine de tout ce qui existe en dehors de lui. Autrement dit, le bébé-Dieu est entré en état de manque, compris qu'il y a un monde extérieur, une inaccessibilité des objets. Les parents sont contents dans la mesure où la rage et la fureur prouvent qu'il est bel et bien vivant. C'est donc dans le déplaisir qu'il advient à la vie.

Comment apaiser la colère divine?

La réponse se trouve dans l'étape suivante, celle de la formation du moi par l'expérience du plaisir. C'est la scène où la grand-mère paternelle, arrivée de Bruxelles pour faire la connaissance de sa petite-fille (la famille habitait à Kobé, le père y étant consul de Belgique), donne à Dieu qui hurle sa colère un bâton au chocolat blanc de Belgique. Le plaisir procuré par l'ingestion de cette substance (qu'ailleurs Nothomb qualifie de "théologale") tait le bruit du déplaisir et de ce silence fait émerger une instance psychique qui parle d'une voix interne à la première personne du singulier:

C'est moi! C'est moi qui vis! C'est moi qui parle! Je ne suis pas “i”" ni "lui”, je suis moi! Tu ne devras plus dire "il" pour parler de toi, tu devras dire "je". Et je suis ton meilleur ami: c'est moi qui te donne le plaisir. (2000:30)

La narratrice ajoute que c'est cette journée-là qu'elle est vraiment née. Pas de vrai vie sans moi, pas de moi sans le plaisir qui le fonde: "Moi, c'est le siège du plaisir. Le plaisir, c'est moi" (2000:31).

L'instance psychique vocale centrée sur le moi est la conscience. Mais cette conscience moïque n'est pas la conscience morale surmoïque que Nothomb appelle dans les romans post-2000 "l'ennemi intérieur". Celui-ci se met en place à la sortie de l'enfance et 
sa fonction est de culpabiliser le moi, "de détruire ce qui en vaut la peine, de montrer la décrépitude contenue dans chaque réalité" (Nothomb, 2001:28, 29) - bref, de transformer tout plaisir en déplaisir. La conscience moïque, au contraire, se met en place au début de l'enfance et le moi est le centre d'une conscience assise dans l'expérience du plaisir. Dans et avec le moi, le plaisir fonde la mémoire et investit l'enfant d'une identité: une famille et une nationalité. Car le plaisir fondateur est belge. Ce n'est pas la langue nationale qui fait émerger le moi, l'identité et la conscience, mais le plaisir national du chocolat. Autrement dit, ce n'est pas la négativité d'une structure signifiante qui transforme l'être en individu mais la positivité d'une substance. La conscience, la pensée, l'intelligence s'enracinent donc dans le sensible:

II existe depuis très longtemps une immense secte d'imbéciles qui opposent sensualité et intelligence. C'est un cercle vicieux: ils se privent de volupté pour exalter leurs capacités intellectuelles, ce qui a pour résultat de les appauvrir. [...]

La délectation rend humble et admiratif envers ce qui l'a rendue possible, le plaisir éveille l'esprit et le pousse tant à la virtuosité qu'à la profondeur. C'est une si puissante magie qu'à défaut de volupté, l'idée de volupté suffit. Du moment qu'existe cette notion, l'être est sauvé. Mais la frigidité triomphante se condamne à la célébration de son propre néant. (2000:34)

Ce moi-plaisir enfantin continue d'être appelé Dieu. Mais ce dieu est dans le monde, dans la vie, dans la nature. Dans Le sabotage amoureux, Nothomb affirme qu'être enfant, c'est être (1993:55). L'enfance est dans l'univers fictionnel nothombien la condition ontologique par excellence, car c'est là que l'être vit pleinement et intensément. Évidemment cet état divin du moi-plaisir n'a rien à voir avec l'état divin du commencement. À remarquer que dans la scène du chocolat, il n'est pas question de tubes. Le tube réapparait juste après sous forme d'aspirateur dans un épisode où il question des pouvoirs du langage. Le premier est le pouvoir de faire exister ou, du moins, d'assurer les êtres dans leur existence. Aussi, les deux premiers mots conventionnellement prononcés par l'enfant, "maman" et "papa", déclenchent chez les parents des réactions de joie et de ravissement qui portent à croire qu'ils n'étaient pas sûrs de l'être et qu'ils avaient besoin d'en avoir la confirmation nominale. Le second pouvoir du langage est d'anéantir et il est attaché à l'aspirateur qui est aussi le troisième mot que l'enfant a choisi de dire. Cet électroménager a pour elle une signification métaphysique: "l'appareil avalait les réalités matérielles qu'il rencontrait et il les transformait en inexistence. II remplaçait le quelque chose par le rien: cette substitution ne pouvait être qu'œuvre divine" (p.40). Lorsqu'elle rencontre l'aspirateur, l'enfant que l'on appellera désormais Amélie, bien que son prénom n'apparaisse jamais dans le roman, "se souvient 
vaguement d'avoir été Dieu il y a peu de temps avant". Elle s'identifie à l'aspirateur comme à un frère car la fonction d'anéantissement de cet appareil réactive "une grande voix" entendue dans sa tête "qui la plongeait dans d'incalculables ténèbres" (2000:40) et qui lui disait: “Rappelle-toi! C'est moi qui vit en toi!". C'est la voix ténébreuse de Dieu le tube retrouvée en écho dans le bruit de cet autre tube qu'est l'aspirateur. C'est la tentation de la mort et la racine de ce qui sera la voix de "l'ennemi intérieur".

La divinité de l'enfant moïque ne consiste pourtant pas à anéantir ce qu'il y a mais à en jouir. La centralité du moi se manifeste dans le culte de l'enfant-idole dont le temple est le splendide jardin nippon, où la petite Amélie passe la journée "au cœur de la beauté et de l'adoration" (200:57). C'est dans le jardin qu'elle apparait, au sens épiphanique du mot, à ses fidèles, notamment à sa gouvernante Nishio-san qui l'idolâtrait (p.58). Sa place centrale dans le jardin est posée en termes d'idolâtrie pour rendre compte de la toute-puissance imaginaire du moi qui se croit un comble ontologique: petit dieu païen qui règne au cœur de la nature printanière, s'identifiant à sa beauté et à sa force vitale et croyant à la stabilité et à pérennité de l'être.

L'aire géographique de la croyance en moi atteignait son plus haut degré de densité dans le jardin. Les murs élevés et chapeautés de tuiles japonaises qui le cloîtraient me dérobaient aux regards des laïcs et prouvaient que nous étions dans un sanctuaire.

Quand Dieu a besoin d'un lieu pour symboliser le bonheur terrestre, il n'opte ni pour l'île déserte, ni pour la plage de sable fin, ni pour le champ de blé mûr, ni pour l'alpage verdoyant: il élit le jardin.

Je partageais son opinion:il n'y a pas meilleur territoire pour régner. Fieffée du jardin, j'avais pour sujets des plantes qui, sur mon ordre, s'épanouissaient à vue d'œil. C'était le premier printemps de mon existence et je n'imaginais pas que cette adolescence végétale connaîtrait un apogée suivi d'un déclin.

Un soir, j'avais dit, à une tige surmontée d'un bouton: "Fleuris". Le lendemain, c'était devenu une pivoine blanche en pleine déflagration. Pas de doute, j'avais des pouvoirs. J'en parlais à Nishio-san qui ne démenti pas.

Depuis la naissance de ma mémoire, en février, le monde n'avait cessé d'éclore. La nature s'associait à mon avènement. (2000:60).

La subjectivation fera chuter ce leurre. Une telle "croyance en moi" situe l'enfant au stade du miroir: "J'étais le centre géométrique d'un cercle de splendeur qui ne cesse de s'élargir" (2000:71). Dans le jardin l'enfant contemple "son avènement", en s'identifiant à l'image idéale de l'être qui jouit de sa plénitude. Une phrase pourrait résumer ce stade: il n'y a pas de manque dans l'être. En langage psychanalytique cela s'appelle la phase phallique 
dans laquelle les enfants croient à l'universalité du phallus (tout être en serait pourvu). Le registre religieux des chapitres consacrés à l'enfant-idole indique que la plénitude ontologique relève de la croyance et souligne la nature imaginaire du moi. Déjà, au sein même du jardin, quelque chose contrarie cette ontologie. La seconde gouvernante, Kashima-san, "est un problème logique dans cette apologétique" (2000:61). Elle constitue une aporie dans le narcissisme enfantin et signifie au moi les limites de son pouvoir (2000:64). Pourquoi? Parce qu'elle renonce aux plaisirs (2000: 63) et, ce faisant, elle refuse de se plier au culte de l'enfant-idole. Ce qui est posé en termes logiques dans cette séquence, sera posé en termes éthiques à la fin du roman, lorsque la méchanceté de Kashima-san l'empêche de faire quoi que ce soit pour sauver l'enfant de la noyade. C'est là une touche de ce qui est développé dans d'autres romans de Nothomb, notamment dans Catilinaires: l'idée que le mal (l'indifférence) est coextensif au vide, qu'il s'enracine dans la négation du plaisir et que le plaisir protège de la tentation de la mort. Or, la négation du plaisir est représentée par le tube qui creuse dans l'être la rigidité inorganique du non-être.

\section{La jouissance aquatique de l'être et la voix méconnaissable du père}

La jouissance de l'être passe par les plaisirs sensoriels - le vert du lac, l'odeur du jardin, le goût de l'alcool de prune - mais sa substance essentielle est l'eau. Pendant la saison humide, la petite Amélie passe la journée dans le petit lac vert où elle se baigne sous l'averse jusqu'à se fondre avec l'univers:

Je plongeais dans le lac et n'en sortais plus. Le moment le plus beau était l'averse: je remontais alors à la surface pour faire la planche et recevoir la sublime douche perpendiculaire. Le monde me tombait sur le corps entier. J'ouvrais la bouche pour avaler sa cascade, je ne refusais pas une goutte de ce qu'il avait à m'offrir. L'univers était largesse et j'avais assez de soif pour le boire jusqu'à la dernière gorgée. (2000:109)

L'eau est l'élément par lequel le corps acquiert la dimension de l'univers et se fond avec lui dans un sentiment aquatique. L'eau est l'élément du moi: "L'eau en dessous de moi, l'eau au-dessus de moi, l'eau en moi - l'eau, c'était moi. Ce n'était pas pour rien que mon prénom, en japonais, comportait la pluie" (2000:109).

C'est dans l'eau que l'enfant frôle la mort par deux fois. La première dans la mer du Japon. Les spectateurs nippons la regardent couler sans rien faire pour la sauver, selon ce que détermine le code d'honneur. La seconde noyade, dans le bassin aux carpes. Et de 
même Kashima-san la regarde mourir sans tenter de la sauver. L'eau met en continuité le moi et le monde mais aussi la jouissance et la mort.

Le récit de la jouissance aquatique relie deux épisodes centrés sur le personnage du père. II y est question de l'énigme que son métier constitue pour l'enfant. Le métier de $\mathrm{M}$. Nothomb est on ne peut plus symbolique. Il est consul de Belgique à Kobé, il exerce donc la fonction de représentation en sa pureté formelle. L'enfant ne comprend pas en quoi consiste la fonction symbolique de consul et son rapport au père l'ignore radicalement. Dans ces deux épisodes le rapport au père passe par la médiation de sa voix, laquelle n'est pas reconnue comme la sienne mais au contraire comme une voix méconnaissable et étrangère au père. Dans le premier épisode, le père chante le nô et les sons bizarres et effrayants qu'il émet changent sa voix en "une plainte méconnaissable" qui épouvante l'enfant et ensuite l'ennuie. Le second épisode où la voix se sépare du père est celui où il tombe dans le caniveau ouvert. Tout d'un coup, l'enfant se rend compte dans l'angoisse que son père n'est pas à ses côtés. Il avait été aspiré par l'égout et la voix qui en sort "venait d'outre-tombe" et "semblait traverser un monde". La voix apparait ainsi comme un objet séparé et autonome, étrange et étranger (au père et au monde), qui cause l'angoisse parce qu'il brise la consistance de la réalité, en y introduisant un trou, une absence: "Les êtres étaient-ils des choses si précaires que l'on puisse les perdre sans motif et sans explication?" (2000:103). Croyant alors avoir compris que son père était égoutier et travaillait dans les canalisations, Amélie le laisse dans l'égout et va jouer. II n'en sera retiré que quelques heures plus tard et son émergence des eaux sales et malodorantes, dite du "Belge Anadyomène", couvre son statut symbolique de matière fécale. Croire, comme elle le fait, que c'était aussi dans l'égout que son père répétait le nô revient à réunir les deux phénomènes vocaux dans le tube mais un tube qui, loin d'être vide, est plein de souillures. Ce tube n'est pas métaphysique. L'égout n'est pas l'aspirateur. Le moi n'accède pas à saisir la fonction symbolique exercée par le père. La disparition du grand corps paternel ne l'élève pas, aux yeux de sa fille, à l'élégance formelle de la condition de pur représentant (de son pays). L'objet vocal, corrélatif de son absence, devient le déchet qui manifeste de retour de sa présence massive au monde; la voix qui n'est pas de ce monde est réintégrée au grand corps immonde du "bonhomme de boue" qui sort du caniveau.

Ce n'est donc pas l'égout qui introduit le manque dans l'être. Cette fonction structurante de la subjectivité est dévolue aux bouches d'égout des carpes.

\section{La subjectivation}

\section{Les carpes et le non-être comme différence sexuelle}


Si l'hérésie de Kahima-san ne faisait que contrarier le moi, le premier contact avec les carpes le trouble et ébranle ses convictions. Avant de voir les carpes, Amélie en entend parler comme du symbole des garçons dans la culture japonaise. Elle apprend qu'il n'y a pas de symbole pour les filles et en est déçue. Les carpes signalent donc une différence pas comme les autres: la différence sexuelle ne se traduit pas en couple d'oppositions (symbole masculin, symbole féminin) mais en une pure différence privée de contenus a priori, autrement dit une pure négativité (le symbole unique creuse la différence en tant que négatif). Aussi, les carpes assument-elles la dimension sexuelle du négatif. Elles signalent que l'être sexué est marqué du sceau du non-être, l'être destiné à la mort. Depuis Hygiène de l'assassin, la sexuation est, dans l'univers nothombien du XXe siècle, identifiée à la déchéance, à l'érosion et corrosion de l'être dès que l'entrée dans la puberté, en rendant le corps apte à la procréation, le soumet irréversiblement à "la vie hormonale". Prétextat tue Léopoldine le jour de ses premières règles; Émile retraité s'isole dans la Maison avec Juliette dont il nie constamment le vieillissement pour relayer l'autosuffisance idyllique de leur amour d'enfance interrompu par l'âge adulte; Omer prive Hazel de son image spéculaire pour en faire un reflet d'Adèle suicidée; Épiphane tue Éthel pour qu'elle n'existe que par son souvenir à lui; Celsius fige la splendeur de Pompéi - autant de stratégies pour "échapper au temps et à toutes les glues que charrie la durée" (1993:93) dont la première est la glu sexuelle. Dans ces couples, y compris Celsius et la ville, le personnage masculin projette son image idéale sur le féminin, supprime l'altérité de celui-ci, le recrée à l'image de son moi, en fait l'objet et l'instrument d'un projet de suppression de la différence sexuelle (ce qui ne veut pas dire forcément absence d'érotisme car il y a chez Nothomb un érotisme enfantin sans sexe qui consiste dans la jouissance du froid).

Les carpes donnent donc à voir la convergence du sexe et de la mort. S'il y a un côté heideggérien chez Nothomb, Sein und Zeit se décline en "différence sexuelle et être-pour-lamort". Quand elle voit des carpes pour la première fois, Amélie est fascinée de leur laideur, de leur obésité, de leur décrépitude: "Etre centenaire pour une carpe, c'était se vautrer dans une durée adipeuse, c'était laisser moisir sa chair vaseuse de poisson d'eau stagnante. II y a encore plus dégoûtant que la jeune graisse: c'est la vieille graisse". (2000:85). Voir les carpes revient à constater que le temps introduit du manque dans l'être, que ce manque est visible dans la graisse ${ }^{2}$ et que, étant donné que les carpes signifient la différence sexuelle, le

\footnotetext{
${ }^{2}$ L'obésité est chez Nothomb la face visible et substantiel du néant, de l'inertie du tube. Le couple Bernardin figure ce rapport à merveille. L'époux, dont la régularité larvaire des visites taciturnes cause un ennui insupportable chez Émile et Juliette, 'était d'autant plus vide qu'il était gros: comme il était gros, il avait plus de volume pour contenir son vide' (1994:82-3). II est comparé aux courges géantes, aux soufflés au fromage et aux discours d'inauguration - 'enflés à proportion de leur vacuité'. L'épouse est ainsi décrite: 'Un kyste, cette chose était un kyste. Eve fut tirée d'une côte d'Adam. Madame Bernardin avait sans doute poussé comme un kyste dans le ventre de notre tortionnaire. Parfois, on opère des malades d'un kyste interne qui pèse le double, voir le
} 
manque dans l'être est d'ordre sexuel. Le dégoût des carpes est le dégoût du sexe ${ }^{3}$ en tant que mort contenue dans la vie. La phase suivante le confirme en montrant que le dégoût fondateur éprouvé par Amélie, quand elle nourrit les carpes à midi, s'étaye en répugnance de la chose sexuelle fantasmée dans l'angoisse nocturne.

\section{Les carpes dans le jardin et la fondation de la subjectivité}

Mais cette constatation qu'il y a du négatif au sein de l'être, ce savoir appris à l'arboretum du Futatabi, reste au-dehors du jardin-sanctuaire et ne concerne pas le moi. Ce n'est que quand les carpes sont introduites dans le jardin en faisant tache sur sa beauté splendide que le moi est vraiment atteint dans son être. L'entrée des carpes dans le jardin cause l'expulsion de l'enfant divin: le moi est chassé du paradis de la plénitude ontologique, il perd la jouissance de l'être, il est dédivinisé. Du moi chassé du paradis résulte le sujet. C'est ce en quoi consiste "l'aventure fondatrice".

Tout commence par un malentendu. Alors qu'Amélie désirait un éléphant en peluche pour son troisième anniversaire et l'avait discrètement signifié à ses parents, ceux-ci, persuadés qu'elle s'intéressait aux carpes, lui en ont offert trois. L'enfant éprouve une déception sans pareille. De plus, les parents lui proposent de baptiser les carpes de son prénom et de ceux de sa sœur et de son frère. Pour parer à cette identification répugnante elle leur donne en alternative les prénoms de la sainte famille: Jésus, Marie, Joseph. La déception cuisante qui est à l'origine de l'aventure fondatrice est ainsi parodiquement mise à l'enseigne du rapport enfant-parents. En effet, la mutation du moi en sujet a comme cause immédiate une opacité dans la demande adressée aux parents qui n'ont pas compris le désir qui y était sous-entendu. Se rendre compte que les parents sont incapables de lui assurer l'objet de son désir, l'éléphant blanc en peluche, ouvre la voie tragique de la subjectivation, celle-ci consistant précisément dans la perte de la jouissance.

Pour satisfaire à la demande des parents, Amélie devait nourrir les poissons tous les jours, tâche qui la dégoûtait à un point inexprimable. Elle raconte ce qu'elle appelle son calvaire dans le registre évangélique, lequel d'ailleurs traverse toute la séquence: après la sainte famille, le sacrifice christique de la fille.

\footnotetext{
triple de leur poids: Palamède avait épousé l'amoncellement de chair dont on l'avait libéré' (1994, p.66). Cette description n'est pas seulement une hyperbole visant à dire que cette femme est une accumulation de matières graisseuses. II s'agit de signifier la consubstantialité et la ressemblance de mari et femme, obèses et taciturnes tous les deux, et provoquant chez Émile et Juliette le même sentiment de dégoût que les carpes causent chez la petite Amélie de trois ans. La répugnante obésité de Bernadette est effectivement posée comme la réduction du corps à la bouche, soit un tube: 'Ce ne fut rien comparé à la répulsion qui me crispa les mâchoires quand le verre s'inséra dans sa bouche. L'orifice replia ce qui lui servait de lèvres et se mit à aspirer. Le lait fut sucé en un seul coup, mais avalé en plusieurs fois; chaque déglutition faisait le bruit d'une ventouse en caoutchouc en train de déboucher un évier. J'étais horrifié. Vite, parler, dire quelque chose' (idem, p.68)

${ }^{3}$ II ne s'agit pas de sexe au sens courant d'activité génitale mais au sens logique de différence sexuelle en tant que pure différence ou sexuation.
} 
Chaque jour, à midi, au moment où le soleil était au plus haut dans le ciel, je pris l'habitude de venir nourrir la trinité. Prêtresse piscicole je bénissais la galette de riz, la rompais et la lançais à la flotte en disant: - Ceci est mon corps livré pour vous. (2000:135)

La parodie du texte évangélique garde la tension entre le comique et le tragique ${ }^{4}$. Donner un cadre christique à la subjectivation n'est peut-être pas sans signification. Si la narratrice cesse d'appeler le personnage "dieu" ou “idole" n'est-ce pas pour signifier que la différence entre moi et sujet prolonge en quelque sorte la différence entre paganisme et christianisme?

Gueules ouvertes pour prendre les morceaux de galette, les carpes montrent au grand jour leurs tubes digestifs et sont alors décrites comme des tubes, leurs corps se réduisant aux bouches d'égout, bouches béantes formant un trou. Ce trou interne mais visible est entouré de la substance organique répugnante aperçue pour la première fois dans l'arboretum. Aussi, les carpes présentent-elles une topologie de bord où inorganique et organique, vide et substance, métaphysique et biologique, sont l'endroit et l'envers.

Le diamètre de leur orifice était presque égal au diamètre de leur corps, ce qui eût évoqué la section d'un tuyau, s'il n'y avait pas eu leurs lèvres poissonneuses qui me regardaient de leur regard de lèvres, ces lèvres saumâtres qui s'ouvraient et se refermaient avec un bruit obscène, ces bouches en forme de bouées qui bouffaient ma bouffe avant de me bouffer moi! (2000:136)

La galette de riz est la chair même du moi, sa substance, son être. En nourrissant les carpes, c'est sa chair qu'elle donne en pâture, elle sacrifie sa vie, comme le Christ. Cet évidemment se traduit concrètement dans l'amaigrissement de l'enfant qui perd le goût de la vie: "j'avais de plus en plus l'impression que c'était ma propre chair qui nourrissait les carpes. Je maigrissais." (p.138). Les carpes-tubes aspirent l'être du moi.

Les carpes ont changé le jardin en un lieu de déplaisir. La narratrice commente que la portée fondatrice de ce dégoût tient au fait qu'il ne s'agit pas d'un dégoût comme ces abjections qui sont le lot des petits enfants curieux. C'est un dégoût au-delà des autres,

\footnotetext{
${ }^{4}$ La réécriture de passages bibliques suit la démarche générale de l'écriture nothombienne qui se tient et se déploie dans une tension constante entre sublimation et désublimation, tragique et comique: la mise en place d'un idéal ou d'une valeur est pénétrée par l'humour et l'ironie sans pour autant tomber dans un registre cynique ou sordide ou dans la raillerie. Le comique intervient dans le sérieux sans que le rire ne dissipe pas ce dont il rit mais, au contraire, l'illumine et le localise (Zupancic 2002:68). Par conséquent la parodie du texte biblique consiste dans une tension entre apologétique et blasphème.
} 
anormal, excentrique, extravagant et qui justement à ce titre fonde la singularité radicale de tout un chacun:

II m'arrive de penser que notre unique spécificité individuelle réside en ceci: dis-moi ce qui te dégoûte et je te dirai qui tu es. Nos personnalités sont nulles, nos inclinations plus banales les unes que les autres. Seules nos répulsions parlent vraiment de nous. (2000:137).

\section{La structure extime du sujet}

Le dégoût fondateur est structurant de la subjectivité et les carpes-tubes sont l'objet dont se soutient sa négativité. Le sujet est l'effet de l'évidement de la substance jouissante du moi (sa chair). Le sujet est non-être. Aussi, n'est-il pas au centre du jardin mais décentré au bord de l'étang sur le point de faillir du dégoût de reconnaître son non-être dans le tube digestif des carpes. II se soutient de l'objet insoutenable et pathologique. Les carpes-tubes sont le contour organique, gras et décrépit du rien du commencement: l'ex-nihilo d'où procède toute créature. Le dégoût prend alors une signification métaphysique formulée dans la réécriture de topoi bibliques:

Et toi, que crois-tu être d'autre? Tu es un tube sorti d'un tube. Ces derniers temps, tu as eu l'impression glorieuse d'évoluer, de devenir de la matière pensante. Foutaise. La bouche des carpes te rendrait-elle si malade si tu n'y voyais ton miroir ignoble? Souviens-toi que tu es tube et que tube tu redeviendras. (2000:145)

On mesure l'écart qui sépare le jardin, en tant qu'image idéale du moi, du miroir ignoble que sont les carpes pour le sujet. La présence des carpes empêche le moi de se projeter sur les objets plaisants qui l'entouraient en trouant irréversiblement la beauté du jardin. Les carpes négativisent le goût de la vie en dégoût, ce qui est le corrélat du sujet sans substance, sans chair et sans image spéculaire. Les carpes sont la présence massive du non-être du sujet. S'y reconnaître ne mène pas le sujet à une identification unificatrice et jubilatoire, car les carpes ne sont pas une image spéculaire mais un regard répulsif qui externalise et donne à voir le noyau dur de pure négativité du sujet. La structure subjective est ainsi une extimité, pour reprendre une notion lacanienne: “(...) ce qui m'est le plus intime est justement ce que je suis contraint de ne pouvoir reconnaître qu'au-dehors" (Lacan, 2006:225; cf.249). Le plus interne, le plus intime, ce point inorganique d'ex-nihilo qui est au cœur du sujet et dont il préfère ne rien savoir, apparait à l'extérieur non comme image mais comme objet radicalement impersonnel, un ça qui n'est pas moi. La mise en place de cette 
structure signale la sortie de l'enfance mythique, le désenchantement précoce de l'enfant qui perd la jouissance imaginaire de l'être (illusion de toute-puissance et de pérennité) et accède au dégoût du sexe fantasmé dans l'angoisse:

La nuit, dans mon lit, je peuplais l'obscurité de bouches béantes. Sous mon oreiller, je pleurais d'horreur. L'autosuggestion était si forte que les gros corps écailleux et flexibles me rejoignaient entre les draps, m'étreignaient - et leur gueule lippue et froide me roulait des pelles. J'étais l'impubère amante de fantasmes pisciformes (...) Ce n'était pas son estomac qui me dégoûtait, mais sa bouche, le mouvement de valvule de ses mandibules qui me violaient les lèvres pendant des éternités nocturnes. (2000:138-9)

La paradoxale structure extime signale encore que le sujet advient dans sa destitution ontologique même et est donc voué au suicide. Les carpes soutiennent le sujet en tant que vide, dans son ontologie négative, dans sa néantisation, ce qui revient à se suicider. Aspirée par le tube, au comble du pathos, Amélie se laisse tomber dans le bassin. Tête cassée, elle se sent redevenir tube et la troisième personne du singulier dépossède la première. "La chose de moins en moins vivante se sent redevenir le tube qu'elle n'a peutêtre jamais cessé d'être". Sauvée in extremis par Nishio-san, Amélie est transportée à l'hôpital. Elle ne contrarie pas la version officielle bâtie par sa mère, qui réduit "l'aventure fondatrice" à une glissade. Amélie savait que sa chute dans l'étang n'était pas due au hasard d'un accident, mais qu'elle avait une cause bien précise, cependant à trois ans elle ne connaissait pas le mot "suicide". L'enfant a abandonné l'illusion de la jouissance mythique, ayant appris qu'il n'y a pas de vie sans mort, que la mort traverse la vie, que la vie vient de la mort et y revient. L'expérience du négatif qui mine l'existence, de l'assomption subjective du manque dans l'être, a laissé une cicatrice sur la tempe gauche, marque indélébile du réel du négatif. Le creux physique (le traumatisme crânien) signe la condition de manque-à-être du sujet (effet de l'expérience traumatique) qui éclate dans la dernière phrase: "Ensuite, il ne s'est plus rien passé". Cette phrase clôt le roman en introduisant le personnage dans une période creuse, plate, dépourvue d'événements et d'histoires, désactivée, latente, passée dans l'attente et l'ennui, bref dans l'existence négative qui est le lot du sujet.

Après Stupeurs et tremblements et Métaphysique des tubes, l'œuvre romanesque de Nothomb subit une inflexion dont le trait le plus saillant est que le rapport hétérosexuel (Prétextat et Léopoldine, Émile et Juliette, Épiphane et Éthel, etc) est remplacé par le rapport entre deux femmes, selon le modèle infantile Amélie-Elena dans Le sabotage amoureux. Ce modèle n'est repris que dans Stupeurs et tremblements avec Amélie-Fubuki et se déploie en 
Plectrude-Clémence, Blanche-Christa, Pannonique-Zdena. L'enfance perd du relief: elle est absente de la problématique d'Antéchrista et d'Acide Sulfurique. Même dans Journal d'Hirondelle, où Nothomb revient au couple hétérosexuel, il n'y est pas question d'enfance et l'amour ne s'inscrit pas dans la structure du stade du miroir. Cosmétique de l'ennemi, Biographie de la faim et Robert des noms propres focalisent les peines et les impasses de la puberté, sans nostalgie de l'enfance. Le rapport amoureux suit le modèle de la première expérience amoureuse d'Amélie avec Elena qui est très différent des amours idylliques. Au lieu de supprimer l'altérité de la partenaire pour en faire l'image idéale du moi, l'amour est maintenant affrontement à son altérité radicale, au mystère de son désir. Elle est l'ennemie qu'il faut impérativement aimer. J'aime celle qui me fait mal, ma tortionnaire: Élena, Fubuki, Christa, Zdena. Les couples hétérosexuels sont constitués de la Belle (Léopoldine, Juliette, Hazel, Éthel) et de la Bête (Prétextat, Émile, Omer, Épiphane), la Bête se contemplant au miroir de la Belle et croyant ainsi accéder à la jouissance de l'être. Dans les couples homosexuels la Belle est la Bête (ou, dans le cas de Zdena, la Bête est la Belle): aimer c'est affronter un partenaire opaque, voire inhumain, dont la beauté voile le mal, autrement dit le noyau de non-être, le tube. J'ai affaire au tube dans l'autre. Ces romans reprennent en termes éthiques la problématique ontologique de Métaphysique des tubes. Cette structure est gardée en version light même dans les romans qui reviennent aux couples hétérosexuels après 2005.

Les romans autobiographiques de 1999 et 2000 partagent la production romanesque de Nothomb entre une première période, où prédominent des romans du moi (structure spéculaire), et une autre dominée par des romans du sujet (structure extime). Métaphysique des tubes qui raconte une subjectivation comme évidement de l'être dramatise magistralement la différence entre la structure moïque et la structure subjective et occupe dans l'ensemble de l'œuvre une position charnière. 


\section{Bibliographie}

LACAN, Jacques (2006). Le Séminaire XVI. D'un Autre à l'autre. Paris: Seuil. Notномв, Amélie (1992). Hygiène de l'assassin. Paris: Albin Michel. (1993). Le sabotage amoureux. Paris: Albin Michel. (1995). Les Catilinaires. Paris: Albin Michel. (1996). Péplum. Paris: Albin Michel. (1997). Attentat. Paris: Albin Michel. (1998). Mercure. Paris: Albin Michel. (1999). Stupeurs et tremblements. Paris: Albin Michel. (2000). La métaphysique des tubes. Paris: Albin Michel. (2001). Cosmétique de l'ennemi. Paris: Albin Michel. (2002). Robert des noms propres. Paris: Albin Michel. (2003). Antéchrista. Paris: Albin Michel. (2004). Biographie de la faim. Paris: Albin Michel. (2005). Acide sulfurique. Paris: Albin Michel. (2006). Journal d'Hirondelle. Paris: Albin Michel.

ZUPANCIC, Alenka (2002). Esthétique du désir, éthique de la jouissance. Paris: Théétète. 